\title{
Effect of Signal Filtering on Image Quality of Projection-Based Magnetic Particle Imaging
}

\author{
Kazuki Shimada, Kenya Murase \\ Department of Medical Physics and Engineering, Division of Medical Technology and Science, Faculty of Health Science, \\ Graduate School of Medicine, Osaka University, Osaka, Japan \\ Email: murase@sahs.med.osaka-u.ac.jp
}

How to cite this paper: Shimada, K. and Murase, K. (2017) Effect of Signal Filtering on Image Quality of Projection-Based Magnetic Particle Imaging. Open Journal of Medical Imaging, 7, 43-55. https://doi.org/10.4236/ojmi.2017.72005

Received: March 15, 2017

Accepted: June 4, 2017

Published: June 7, 2017

Copyright $\odot 2017$ by authors and Scientific Research Publishing Inc. This work is licensed under the Creative Commons Attribution International License (CC BY 4.0).

http://creativecommons.org/licenses/by/4.0/

cc) (i) Open Access

\begin{abstract}
Purpose: Magnetic particle imaging (MPI) allows for imaging of the spatial distribution of magnetic nanoparticles (MNPs) in positive contrast, with high sensitivity, high spatial resolution, and high imaging speed. It is necessary to increase the signal-to-noise ratio to enhance the reliability of MPI. The purpose of this study was to investigate the effect of signal filtering on the image quality and quantitativity in projection-based MPI using phantoms. Materials and Methods: We fabricated two kinds of phantom (cylindrical tube phantom with a diameter of $6 \mathrm{~mm}$ and A-shaped phantom) and evaluated the effect of signal filtering in terms of root-mean-square (RMS) granularity and the correlation coefficient between iron concentrations of MNPs and average MPI values for four filter modes (THRU, BPF, BEF, and LPF). In the THRU mode, the signal input was output without passing through the filter. In the BPF mode, only the third-harmonic signal was passed using a band-pass filter (central frequency: $1200 \mathrm{~Hz}$, band width: $1 / 3$ octave). In the BEF mode, the first-harmonic signal was eliminated using a band-elimination filter (central frequency: $400 \mathrm{~Hz}$, band width: 1/3 octave). In the LPF mode, only the signal with a frequency less than the third-harmonic frequency was passed using a low-pass filter (cut-off frequency: $1200 \mathrm{~Hz},-24 \pm 2 \mathrm{~dB}$ /octave). The RMS granularity was obtained by calculating standard deviations of the pixel values in the MPI image without MNPs, whereas average MPI values were obtained by drawing a circular region of interest with a diameter of $6 \mathrm{~mm}$ on the MPI image of the cylindrical tube phantom. Results: When using the filtered backprojection (FBP) method with a ramp filter for image reconstruction, the RMS granularity and correlation coefficient decreased in the order of THRU, BPF, BEF, and LPF. In the BPF mode, however, some artifacts were observed. When using the maximum likelihood-expectation maximization (ML-EM) algorithm with an iteration number of 15 , the correlation coefficient decreased in the order of THRU, BPF, BEF, and LPF, whereas the RMS granularity did not largely depend on the filter mode and was significantly $(p<0.05)$ lower than
\end{abstract}


that for the FBP method for all the filter modes. Conclusion: The BEF mode is adequate for the FBP method in projection-based MPI, whereas THRU is a best option in use of the ML-EM algorithm.

\section{Keywords}

Magnetic Particle Imaging (MPI), Magnetic Nanoparticles (MNPs), Signal

Filtering, Image Quality, Root-Mean-Square (RMS) Granularity

\section{Introduction}

In 2005, a new imaging method called magnetic particle imaging (MPI) was introduced [1]. MPI uses the nonlinear magnetization response of magnetic nanoparticles (MNPs) to an alternating magnetic field called the drive magnetic field (DMF) and allows for imaging of the spatial distribution of MNPs in positive contrast, with high sensitivity, high spatial resolution, and high imaging speed [1]. In MPI, spatial encoding is performed by saturating the MNPs over most of the imaged region except in the vicinity of a special position called the field-free point (FFP) [1] or field-free line (FFL) [2] using a static magnetic field called the selection magnetic field. When MNPs are exposed to the DMF, the spectrum of the magnetization response contains not only the excitation frequency but also higher harmonics owing to their nonlinear magnetization curve [1]. When MNPs are located within the FFP or FFL, odd-harmonic signals are observed. When they are located outside the FFP or FFL, odd-harmonic signals are attenuated and alternatively even-harmonic signals are increased. Based on these phenomena, the spatial distribution of MNPs can be imaged by moving the position of the FFP or FFL, while receiving odd-harmonic signals. Since the third-harmonic signal is the largest of the odd-harmonic signals except for the first-harmonic signal, it is commonly used for signal detection in MPI [3].

Recently, we also developed an MPI scanner with an FFL-encoding scheme for small animal studies [3] [4]. To date, we have used our MPI system to image the spatial distribution of MNPs and to quantify the amount of MNPs and their temporal changes in various tissues and organs such as the tumor and lung in mice, and reported that MPI is a useful tool for preclinical studies [5] [6] [7] [8].

In MPI, the small signal generated by the MNPs exposed to the DMF is detected using a receiving coil and then MPI images are reconstructed from the signals obtained. Therefore, it is important to secure the image quality and quantitativity of the resulting MPI images by increasing the signal-to-noise ratio. A serious issue regarding signal detection in MPI is that the signal generated by MNPs is superimposed with the direct feed through interference of the excitation signal derived from the excitation coil generating the DMF, which directly couples with the receiving coil [9] [10]. Graeser et al. proposed the combined use of a band-stop filter and the cancellation technique using a gradiometer coil to improve the MPI image quality [10]. We developed a simple and practical me- 
thod for correcting the inhomogeneous sensitivity of a receiving coil together with the feed through interference using a blank scan [9]. By combining the filtering method adopted by Graeser et al. [10] and our method [9], we would expect that the reliability of our MPI system can be further enhanced. In this study, we investigated the effect of signal filtering with various analog filter modes on the image quality and quantitativity of the MPI images obtained by our projection-based MPI system using phantom studies.

\section{Materials and Methods}

\subsection{Magnetic Particle Imaging System}

Figure 1 shows a photograph of our MPI scanner, which is an extended version (second generation) of our previous MPI scanner [3] [4]. Figure 2 shows a block diagram for data acquisition and processing in our MPI system. The details of our MPI system are described in our previous papers [3] [4]. In brief, the FFL was generated by two opposing neodymium magnets. The gradient strength was 3.9 T/m in our second-generation MPI scanner. An excitation coil for generating the DMF and a receiving coil were placed between the two neodymium magnets. AC power for generating the DMF was supplied by a programmable power supply (EC1000S, NF Co., Yokohama, Japan) and controlled with a sinusoidal wave generated using a digital function generator (DF1906, NF Co., Yokohama, Japan). The frequency and peak-to-peak strength of the DMF were taken as 400 $\mathrm{Hz}$ and $20 \mathrm{mT}$, respectively. The signal generated by MNPs was detected by a gradiometer-type receiving coil and transported to a multifunction filter (3611, NF Co., Yokohama, Japan) (highlighted in yellow in Figure 2). The signal was amplified by $20 \mathrm{~dB}$ (10 times) and filtered by this multifunction filter. Then, the third-harmonic signal was extracted using a lock-in amplifier (LI5640, NF Co., Yokohama, Japan). The output of the lock-in amplifier was converted to digital data by a personal computer (PC) with a data acquisition device with a universal serial bus port (USB-6212, National Instruments Co., TX, USA). The sampling duration and total sampling time were taken as $100 \mu$ s and $10 \mathrm{~ms}$, respectively.

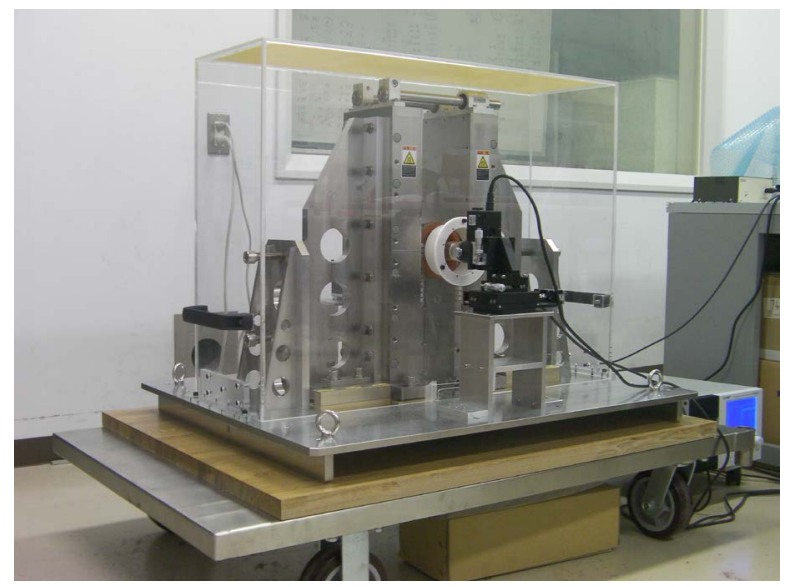

Figure 1. A photograph of our second-generation scanner for magnetic particle imaging (MPI). 


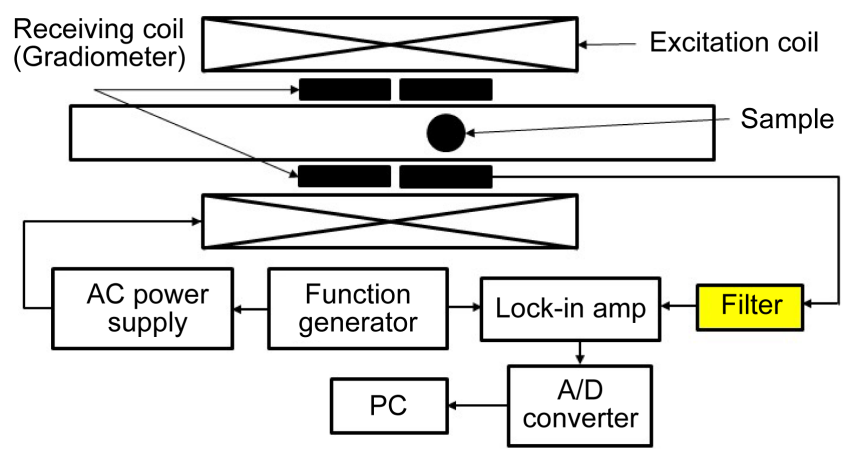

Figure 2. A block diagram for data acquisition and processing in our MPI system. The multifunction filter targeted in this study is highlighted in yellow.

In order to acquire projection data for image reconstruction, a sample (phantom) placed in the receiving coil was rotated over $180^{\circ}$ at a sampling angle of $5^{\circ}$ and translated from -16 to $16 \mathrm{~mm}$ in the $\mathrm{x}$-direction at $1 \mathrm{~mm}$ intervals using an XYZ-axes rotary stage (HPS80-50X-M5, Sigma Koki Co., Tokyo, Japan), which was controlled using LabVIEW (National Instruments Co., TX, USA). Each set of projection data was transformed into 64 bins by linear interpolation and image reconstruction was performed using the filtered back-projection (FBP) method with a ramp filter [11] as a reconstruction filter or the maximum likelihood-expectation maximization (ML-EM) algorithm with an iteration number of 15. The details of the FBP method and the ML-EM algorithm are described in our previous paper [3]. When using the FBP method or ML-EM algorithm for image reconstruction, preprocessing was not performed. In this study, we defined the MPI value as the pixel value of the MPI image reconstructed from the third-harmonic signals.

\subsection{Magnetic Nanoparticles and Phantoms}

We used Resovist ${ }^{\oplus}$ (Fujifilm RI Pharma Co., Tokyo, Japan) as the source of MNPs in this study. Resovist ${ }^{\oplus}$ is composed of MNPs (maghemite, $\gamma-\mathrm{Fe}_{2} \mathrm{O}_{3}$ ) coated with carboxydextran and is an organ-specific contrast agent for magnetic resonance imaging (MRI). We prepared samples with iron concentrations of 0 , 100,250 , and $500 \mathrm{mM}^{\text {Resovist }}{ }^{\oplus}$ to investigate the relationship between the iron concentration of MNPs and the MPI value. It should be noted that because the molar mass of iron $(\mathrm{Fe})$ is $55.8 \mathrm{~g} / \mathrm{mol}$, iron concentrations of 100,250 , and 500 $\mathrm{mM}$ correspond to $5.6,14.0$, and $27.9 \mathrm{mg} \mathrm{Fe} / \mathrm{mL}$, respectively. The adjustment of the iron concentration was performed with saline.

For phantom studies, we made two kinds of phantom as illustrated in Figure 3 ; one was a cylindrical polyethylene tube phantom (6 $\mathrm{mm}$ in inner diameter, 8 $\mathrm{mm}$ in outer diameter, $5 \mathrm{~mm}$ in length, and $100 \mu \mathrm{L}$ in volume) filled with Resovist $^{\oplus}$ having one of the above four iron concentrations (Figure 3(a)); the other was an A-shaped phantom consisting of silicon tubes $2 \mathrm{~mm}$ in inner diameter and $3 \mathrm{~mm}$ in outer diameter and filled with Resovist ${ }^{\oplus}$ having an iron concentration of $500 \mathrm{mM}$ (Figure 3(b)). 


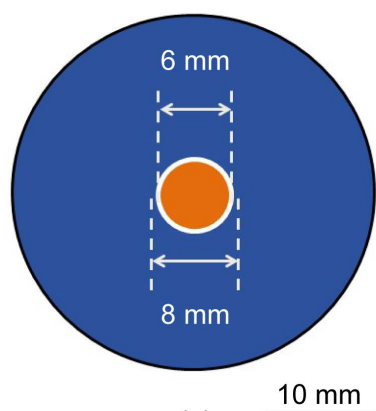

(a)

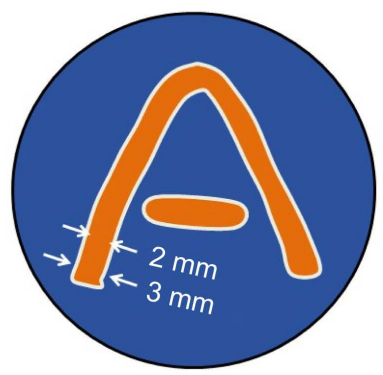

(b) $10 \mathrm{~mm}$

Figure 3. Illustrations of two kinds of phantom used in this study. (a) A cylindrical tube phantom and (b) An A-shaped phantom. Scale bar $=10 \mathrm{~mm}$.

\subsection{Multifunction Filter}

As previously described, the signal generated by MNPs was filtered using a multifunction filter (highlighted in yellow in Figure 2). In this study, we used the following four filter modes in the multifunction filter (3611, NF Co., Yokohama, Japan): THRU, BPF, BEF, and LPF. In the THRU mode, the signal input was output without passing through the filter. In the BPF mode, only the thirdharmonic signal was passed using a band-pass filter (central frequency: $1200 \mathrm{~Hz}$, band width: $1 / 3$ octave). In the BEF mode, the first-harmonic signal was eliminated using a band-elimination filter (central frequency: $400 \mathrm{~Hz}$, band width: 1/3 octave) to suppress the feed through interference from the excitation coil. In the LPF mode, only the signal with a frequency less than the third-harmonic frequency was passed using a low-pass filter (cut-off frequency: $1200 \mathrm{~Hz}$, gain: -24 $\pm 2 \mathrm{~dB}$ /octave). The pass band gain was taken as $20 \mathrm{~dB}$ (10 times) in all the filter modes. Figure 4 illustrates the frequency responses of the above four filter modes used in this study.

\subsection{Data and Statistical Analyses}

Unless specifically stated, data were expressed as the mean \pm standard deviation (SD) for $\mathrm{n}=3$. In phantom studies, we calculated average MPI values by drawing a circular region of interest (ROI) with a diameter of $6 \mathrm{~mm}$ on MPI images of the cylindrical tube phantom. The correlation between the iron concentrations of MNPs and the average MPI values was analyzed by plotting linear regression lines and the correlation coefficients and regression equations were calculated.

We calculated the root-mean-square (RMS) granularity [12] using the MPI images without MNPs $(0 \mathrm{mM})$ to evaluate the image quality. The RMS granularity was calculated by the following equation:

$$
\text { RMS granularity }=\sqrt{\frac{\sum_{i=1}^{N}\left(M P I_{i}-M P I_{m}\right)^{2}}{N-1}},
$$

where $M P I_{i}$ and $M P I_{m}$ denote the MPI value at pixel $i$ and the mean MPI value, respectively, and $N$ is the total number of pixels. In this study, $N$ was taken as 64 $\times 64$. 

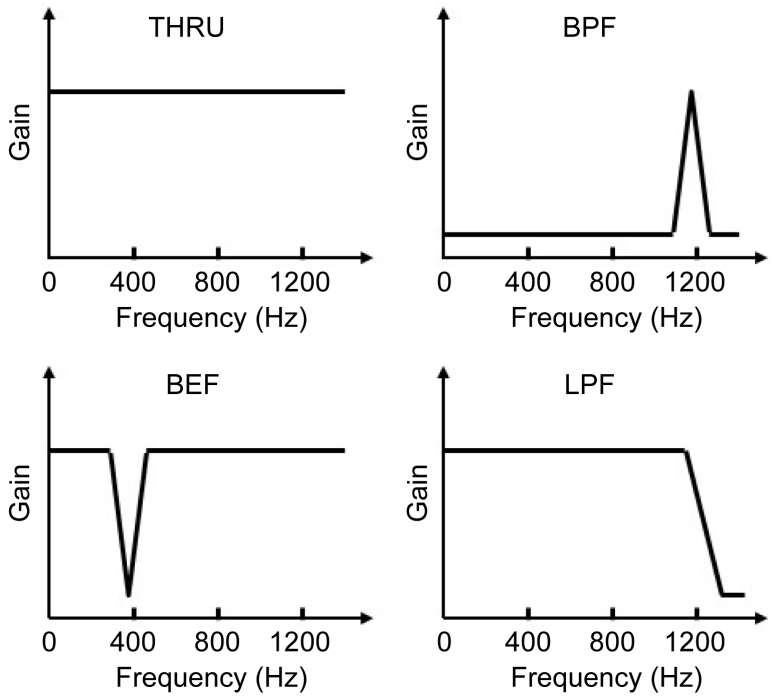

Figure 4. Illustrations of the frequency responses in four filter modes (THRU, $\mathrm{BPF}, \mathrm{BEF}$, and LPF). In the THRU mode, the signal input was output without passing through the filter. In the BPF mode, only the third-harmonic signal was passed using a band-pass filter (central frequency: $1200 \mathrm{~Hz}$, band width: $1 / 3$ octave). In the BEF mode, the first-harmonic signal was eliminated using a band-elimination filter (central frequency: $400 \mathrm{~Hz}$, band width: 1/3 octave). In the LPF mode, only the part of signal with a frequency less than the thirdharmonic frequency was passed using a low-pass filter (cut-off frequency: 1200 $\mathrm{Hz}$, gain: $-24 \pm 2 \mathrm{~dB}$ /octave).

Differences in the RMS granularity among groups were analyzed by one-way analysis of variance (ANOVA). Differences in the average MPI values among the four filter modes were also analyzed by ANOVA. Statistical significance was determined by Tukey's multiple comparison test. When analyzing the difference between two groups, the Student's $t$-test was used. A $p$ value less than 0.05 was considered statistically significant.

\section{Results}

Figure 5 shows the relationship between the iron concentration of MNPs and the average MPI value for the four filter modes shown in Figure 4. Figure 5(a) and Figure 5(b) are the results using the FBP method and the ML-EM algorithm for image reconstruction, respectively. Table 1 summarizes the correlation coefficients and regression equations between the iron concentration of MNPs and the average MPI value in the MPI images reconstructed from the signals processed by the four filter modes (Figure 4) using the FBP method (Figure 5(a)), whereas Table 2 is for the ML-EM algorithm (Figure 5(b)). Although there were excellent linear correlations between the iron concentration of MNPs and the average MPI value and the correlation coefficients exceeded 0.996 in all cases, the correlation coefficient slightly decreased in the order of THRU, BPF, BEF, and LPF (Table 1 and Table 2). When we analyzed the statistical significance in the average MPI value among the four filter modes using ANOVA, there were significant $(p<0.05)$ differences between THRU and BPF, between THRU 


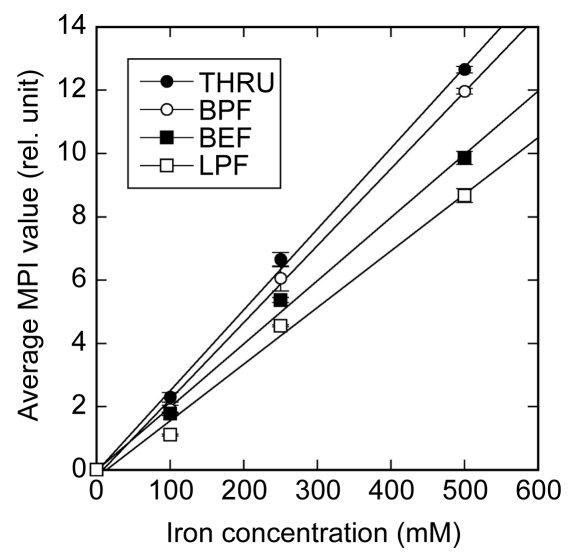

(a)

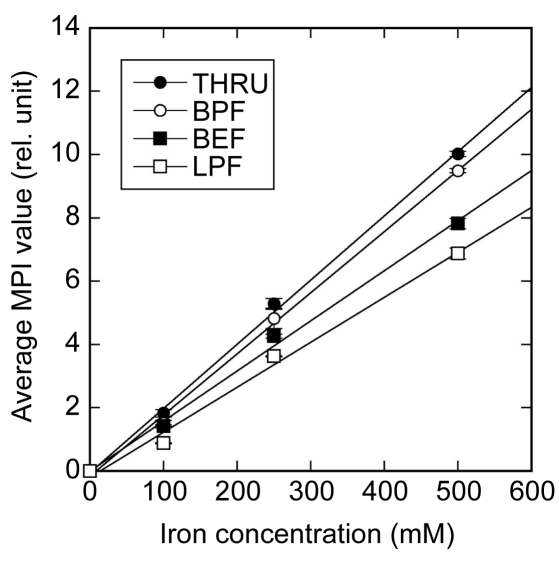

(b)

Figure 5. Relationship between the iron concentration of magnetic nanoparticles (MNPs) and the average MPI value for four filter modes (THRU, BPF, BEF, and LPF) (a) when the filtered back-projection (FBP) method with a ramp filter was used for image reconstruction and (b) when the maximum likelihood-expectation maximization (ML-EM) algorithm with an iteration number of 15 was used. Data are represented by the mean \pm standard deviation (SD) for $\mathrm{n}=3$. The correlation coefficients and regression equations for (a) and (b) are summarized in Table 1 and Table 2, respectively.

Table 1. Summary of the correlation coefficients and regression equations between the iron concentration of magnetic nanoparticles (MNPs) $(x)$ and the average magnetic particle imaging (MPI) value $(y)$ for four filter modes (THRU, BPF, BEF, and LPF). The filtered back-projection (FBP) method with a ramp filter was used for image reconstruction.

\begin{tabular}{ccc}
\hline Filter Mode & Correlation Coefficient & Regression Equation \\
\hline THRU & 0.999 & $\mathrm{y}=0.0256 \mathrm{x}-0.0223$ \\
BPF & 0.999 & $\mathrm{y}=0.0243 \mathrm{x}-0.174$ \\
BEF & 0.998 & $\mathrm{y}=0.0200 \mathrm{x}+0.0165$ \\
LPF & 0.996 & $\mathrm{y}=0.0179 \mathrm{x}-0.208$ \\
\hline
\end{tabular}

Table 2. Summary of the correlation coefficients and regression equations between the iron concentration of MNPs $(x)$ and the average MPI value $(y)$ for four filter modes (THRU, BPF, BEF, and LPF) were used. The maximum likelihood-expectation maximization (ML-EM) algorithm with an iteration number of 15 was used for image reconstruction.

\begin{tabular}{ccc}
\hline Filter Mode & Correlation Coefficient & Regression Equation \\
\hline THRU & 0.999 & $\mathrm{y}=0.0203 \mathrm{x}-0.0212$ \\
BPF & 0.999 & $\mathrm{y}=0.0193 \mathrm{x}-0.144$ \\
BEF & 0.998 & $\mathrm{y}=0.0159 \mathrm{x}+0.00762$ \\
LPF & 0.996 & $\mathrm{y}=0.0142 \mathrm{x}-0.170$ \\
\hline
\end{tabular}

and BEF, between THRU and LPF, between BPF and LPF, and between BEF and $\mathrm{LPF}$ at an iron concentration of $100 \mathrm{mM}$ in both cases (Figure 5). There were significant $(p<0.05)$ differences between THRU and BEF, between THRU and LPF, between BPF and LPF, and between BEF and LPF at an iron concentration of $250 \mathrm{mM}$ in both cases (Figure 5). There were significant $(p<0.05)$ differences for all combinations of the filter modes at an iron concentration of $500 \mathrm{mM}$ in 
both cases (Figure 5).

Figure 6 shows the RMS granularity for the four filter modes shown in Figure 4. Figure 6(a) and Figure 6(b) are the RMS granularities for the FBP method and for the ML-EM algorithm, respectively. As shown by * in Figure 6(a), there were significant $(p<0.05)$ differences between THRU and LPF and between BPF and LPF when analyzed by ANOVA. In contrast, there were no significant differences for any combinations of the four filter modes when using the ML-EM algorithm (Figure 6(b)). When we compared the RMS granularity values between the FBP method and ML-EM algorithm using the Student's $t$-test, the RMS granularity using the ML-EM algorithm was significantly $(p<0.05)$ lower than that using the FBP method for any of filter modes.

Figure 7 shows the MPI images of a 6-mm-diameter cylindrical tube phantom filled with $500 \mathrm{mM}$ Resovist ${ }^{\oplus}$ (Figure 3(a)) for four filter modes (Figure 4). The upper and lower rows show those reconstructed using the FBP method and ML-EM algorithm, respectively. As shown in Figure 7, the MPI value decreased

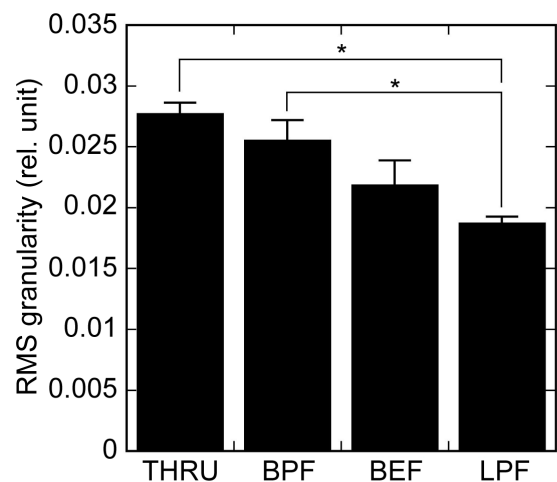

(a)

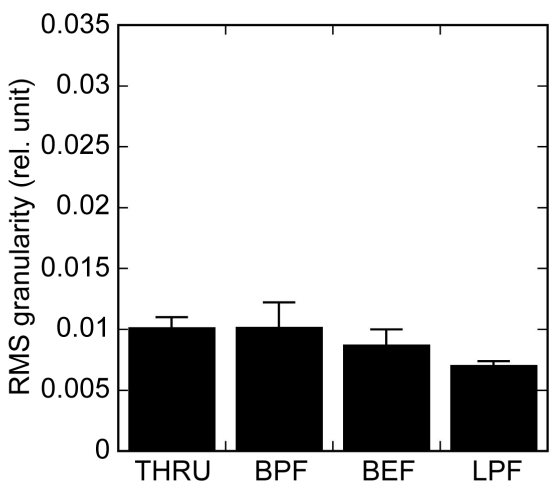

(b)

Figure 6. Root-mean-square (RMS) granularity calculated from Equation (1) for four filter modes (THRU, BPF, BEF, and LPF) (a) when the FBP method was used for image reconstruction and (b) when the ML-EM algorithm was used. Bar and error bar represent the mean and SD for $\mathrm{n}=3$, respectively. ${ }^{*} p<0.05$.
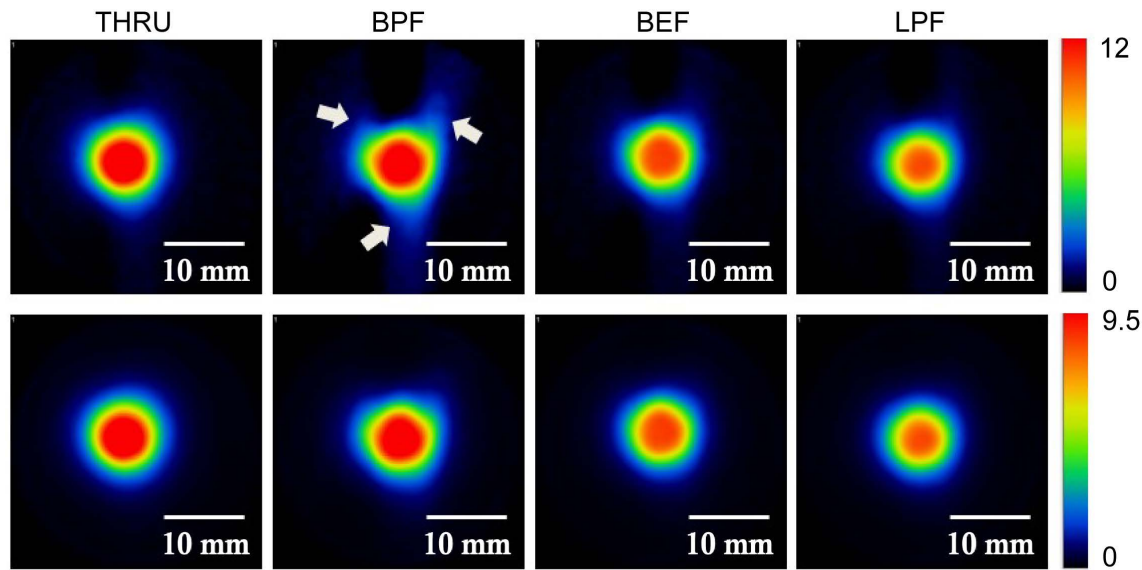

Figure 7. MPI images of a cylindrical tube phantom for four filter modes (THRU, BPF, BEF, and LPF). The upper and lower rows show images when the FBP method and the ML-EM algorithm were used for image reconstruction, respectively. Scale bar $=10 \mathrm{~mm}$. 
in the order of THRU, BPF, BEF, and LPF in both cases. When using the BPF mode, some artifacts were observed in the MPI image reconstructed using the FBP method (shown by arrows in the upper row of Figure 7), whereas such artifacts were reduced in the MPI image reconstructed using the ML-EM algorithm (lower row).

Figure 8 shows the MPI images of the A-shaped phantom filled with $500 \mathrm{mM}$ Resovist $^{\oplus}$ (Figure 3(b)) for four filter modes (Figure 4). The upper and lower rows show the MPI images reconstructed using the FBP method and ML-EM algorithm, respectively. As in Figure 7, the MPI value decreased in the order of THRU, BPF, BEF, and LPF in both cases.

Figure 9(a) shows the horizontal profiles through the center of the MPI image of a 6-mm-diameter cylindrical tube phantom (Figure 3(a)) reconstructed using the FBP method (upper row of Figure 7), whereas Figure 9(b) shows the case when using the ML-EM algorithm (lower row of Figure 7). As shown
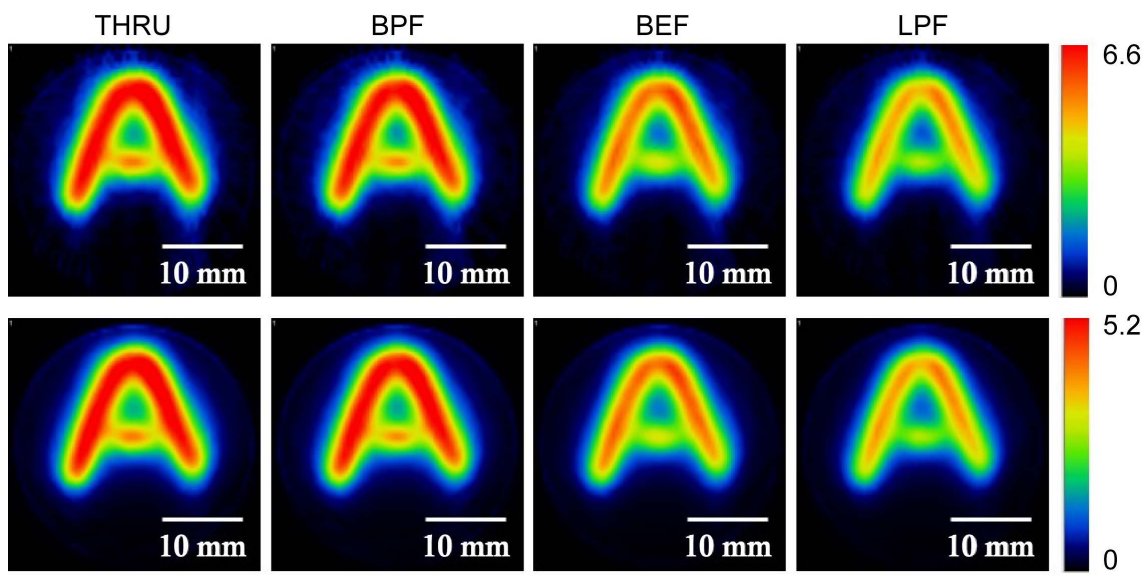

Figure 8. MPI images of an A-shaped phantom for four filter modes (THRU, BPF, BEF, and LPF). The upper and lower rows show images when the FBP method and the ML-EM algorithm were used for image reconstruction, respectively. Scale bar $=10 \mathrm{~mm}$.

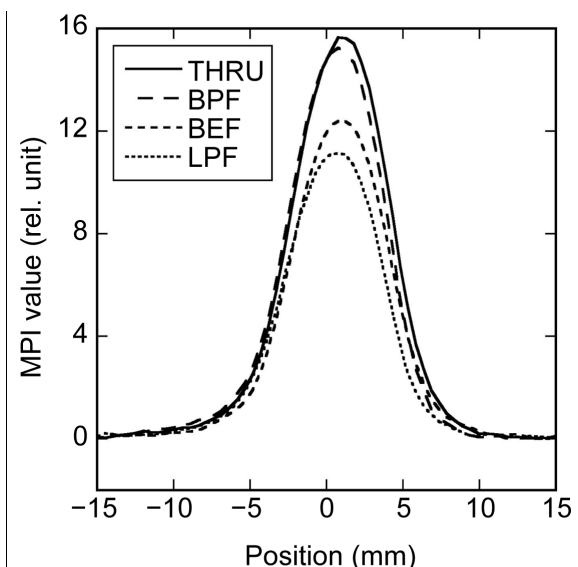

(a)

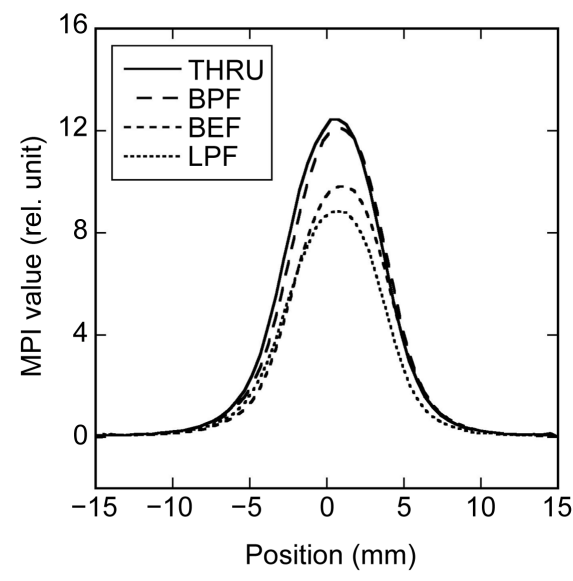

(b)

Figure 9. Horizontal profiles through the center of the MPI image of a cylindrical tube phantom (a) when the FBP method was used for image reconstruction and (b) when the ML-EM algorithm was used. 
in Figure 9, the MPI value at the peak of the profile decreased in the order of THRU, BPF, BEF, and LPF in both cases. When we calculated the full width at half maximum (FWHM) values from the profiles, they were approximately 7.0 $\mathrm{mm}$ and did not largely depend on the filter mode and reconstruction method.

\section{Discussion}

In our projection-based MPI system, spatial encoding is performed by rotating and translating a sample and receiving coil simultaneously, while fixing the FFL, and the third-harmonic signals received by a gradiometer coil are used as projection data for image reconstruction [3] [4]. As shown in Figure 5, there were excellent linear correlations between the iron concentration of MNPs and the average MPI value and the correlation coefficients between them exceeded 0.996 in all cases (Table 1 and Table 2), indicating that the quantitativity of our MPI system is satisfactory. In our MPI system, however, signals with frequencies different from the third-harmonic frequency can become noise and lead to deterioration of the image quality. In particular, a signal with the same frequency as that of the DMF can interfere greatly with signal detection not only in our MPI system but also in others, and thus it is important to eliminate this interference in order to secure the reliability of MPI [9] [10]. Although our MPI system adopted a lock-in amplifier for extracting the third-harmonic signals as previously described, it was not sufficient for removing this interference completely [9].

As pointed out by Graeser et al. [10], when using the cancellation technique with a gradiometer, it is essential that the cancellation signal received by the gradiometer coil has the same phase and amplitude as the induced excitation signal. To achieve this, however, is challenging. Although we also adopted the cancellation technique with a gradiometer coil for eliminating the direct feed through interference from an excitation coil, it was difficult to eliminate this interference completely using this approach alone [9]. Thus, in this study, we considered the combination of the lock-in amplifier, cancellation technique, and analog filtering method, and investigated the feasibility of this approach to improve the image quality in projection-based MPI using phantoms.

When using the FBP method for image reconstruction, the use of the THRU mode showed the largest RMS granularity (Figure 6(a)), whereas the correlation coefficient between the iron concentration of MNPs and the average MPI value was the highest (Table 1). Although the BPF mode showed almost the same results, some artifacts were observed in the MPI image (shown by arrows in the upper row of Figure 7), indicating that the use of BPF may deteriorate the distribution of the receiving coil sensitivity. When we eliminated the signal with a specific frequency using BEF or LPF, the RMS granularity decreased and the image quality was slightly improved (Figure 6(a), Figure 7, and Figure 8). However, the correlation coefficient between the iron concentration of MNPs and the average MPI value slightly decreased. In the BEF mode, it appears that the RMS granularity is improved because the feed through interference from the 
DMF is reduced. As shown in Figure 5(a), however, the average MPI value for the BEF mode was significantly $(p<0.05)$ lower than that for the THRU mode at any of iron concentrations of MNPs, which may lead to slight deterioration of the correlation between the iron concentration of MNPs and the average MPI value (Table 1 ). In the LPF mode, the RMS granularity was significantly ( $p<$ 0.05) lower than those for the THRU and BPF modes (Figure 6(a)). This appears to be due to the reduction of the high-frequency components included in the signal. In contrast, however, the correlation coefficient between the iron concentration of MNPs and the average MPI value was the lowest (Table 1). This appears to be mainly due to the attenuation of the third-harmonic signal, which is confirmed by the fact that the average MPI value for the LPF mode is the lowest at any of iron concentrations of MNPs (Figure 5(a) and Figure 9(a)). When the FBP method is used for image reconstruction in our projection-based MPI system, the BEF mode is recommended.

When the ML-EM algorithm is used for image reconstruction, there were no significant differences in the RMS granularity among all combinations of the four filter modes (Figure 6(b)). If we compared the RMS granularity values between the MPI images reconstructed using the FBP method (Figure 6(a)) and the ML-EM algorithm (Figure 6(b)), the RMS granularity for the ML-EM algorithm was always significantly $(p<0.05)$ lower than that for the FBP method for any of filter modes. The correlation between the iron concentration of MNPs and the average MPI value showed the same tendency as that for the FBP method (Figure 5, Table 1, and Table 2). When the ML-EM algorithm is used for image reconstruction in our projection-based MPI system, the THRU mode is recommended.

As shown in Figure 9, the MPI values at the peaks of the profiles decreased in the order of THRU, BPF, BEF, and LPF for the FBP method and the ML-EM algorithm. To investigate the effect of signal filtering on the spatial resolution in MPI, we calculated the FWHM values from these profiles. As previously described, however, the FWHM values obtained in this study did not largely depend on the filter mode and reconstruction method. This appears to be mainly due to the diameter $(6 \mathrm{~mm})$ of the cylindrical tube phantom used in this study (Figure 3(a)) being too large to evaluate the FWHM precisely. Thus, it would be necessary to use a tube phantom with a smaller diameter to precisely evaluate the effect of signal filtering on the spatial resolution in MPI.

As previously described, for the FBP method, we used a ramp filter [11] as a reconstruction filter, mainly to minimize the effect of the reconstruction filter on the reconstructed MPI image. A Shepp-Logan [13] or Chesler filter [14], however, has generally been used instead of the ramp filter to suppress the high-frequency components included in the projection data. Thus, for the practical application of projection-based MPI, it will also be necessary to study effects of such reconstruction filters.

In this study, we took the iteration number of 15 for the ML-EM algorithm [3] [4]. It is known, however, that the image quality of a reconstructed image using 
the ML-EM algorithm depends on the iteration number [15]. Thus, it will also be necessary to study the dependency of the image quality and quantitativity of the MPI image on the iteration number. These studies are currently in progress.

\section{Conclusion}

In this study, we investigated the image quality and quantitativity of the MPI images reconstructed from the signals processed by various analog filter modes using the FBP method and ML-EM algorithm. Our results showed that the BEF mode is adequate for the FBP method in projection-based MPI, whereas THRU is a best option in use of the ML-EM algorithm.

\section{Acknowledgements}

This work was supported by a Grant-in-Aid for Scientific Research (Grant No. 25282131) from the Japan Society for the Promotion of Science (JSPS).

\section{References}

[1] Gleich, B. and Weizenecker, J. (2005) Tomographic Imaging Using the Nonlinear Response of Magnetic Particles. Nature, 435, 1214-1217. https://doi.org/10.1038/nature03808

[2] Goodwill, P.W., Konkle, J.J., Zheng, B., Saritas, E.U. and Conolly, S.M. (2012) Projection X-Space Magnetic Particle Imaging. IEEE Transactions on Medical Imaging, 31, 1076-1085. https://doi.org/10.1109/TMI.2012.2185247

[3] Murase, K., Hiratsuka, S., Song, R. and Takeuchi, Y. (2014) Development of a System for Magnetic Particle Imaging Using Neodymium Magnets and Gradiometer. Japanese Journal of Applied Physics, 53, Article ID: 067001. https://doi.org/10.7567/JJAP.53.067001

[4] Murase, K., Song, R. and Hiratsuka, S. (2014) Magnetic Particle Imaging of Blood Coagulation. Applied Physics Letters, 104, Article ID: 252409.

https://doi.org/10.1063/1.4885146

[5] Murase, K., Aoki, M., Banura, N., Nishimoto, K., Mimura, A., Kuboyabu, T. and Yabata, I. (2015) Usefulness of Magnetic Particle Imaging for Predicting the Therapeutic Effect of Magnetic Hyperthermia. Open Journal of Medical Imaging, 5, 8599. https://doi.org/10.4236/ojmi.2015.52013

[6] Nishimoto, K., Mimura, A., Aoki, M., Banura, N. and Murase, K. (2015) Application of Magnetic Particle Imaging to Pulmonary Imaging Using Nebulized Magnetic Nanoparticles. Open Journal of Medical Imaging, 5, 49-55. https://doi.org/10.4236/ojmi.2015.52008

[7] Kuboyabu, T., Yabata, I., Aoki, M., Banura, N., Nishimoto, K., Mimura, A. and Murase, K. (2016) Magnetic Particle Imaging for Magnetic Hyperthermia Treatment: Visualization and Quantification of the Intratumoral Distribution and Temporal Change of Magnetic Nanoparticles In Vivo. Open Journal of Medical Imaging, 6, 1-15. https://doi.org/10.4236/ojmi.2016.61001

[8] Kuboyabu, T., Ohki, A., Banura, N. and Murase, K. (2016) Usefulness of Magnetic Particle Imaging for Monitoring the Effect of Magnetic Targeting. Open Journal of Medical Imaging, 6, 33-41. https://doi.org/10.4236/ojmi.2016.62004

[9] Murase, K., Banura, N., Mimura, A. and Nishimoto, K. (2015) Simple and Practical Method for Correcting the Inhomogeneous Sensitivity of a Receiving Coil in Mag- 
netic Particle Imaging. Japanese Journal of Applied Physics, 54, Article ID: 038001. https://doi.org/10.7567/JJAP.54.038001

[10] Graeser, M., Knopp, T., Gruttner, M., Sattel, T.F. and Buzug, T.M. (2013) Analog Receive Signal Processing for Magnetic Particle Imaging. Medical Physics, 40, Article ID: 042303. https://doi.org/10.1118/1.4794482

[11] Ramachandran, G.N. and Lakshminarayanan, A.V. (1971) 3D Reconstructions from Radiographs and Electron Micrographs: Application of Convolutions instead of Fourier Transforms. Proceedings of National Academy of Science USA, 68, 22362240. https://doi.org/10.1073/pnas.68.9.2236

[12] Altman, J.H. (1964) The Measurement of RMS Granularity. Applied Optics, 3, 35 38. https://doi.org/10.1364/AO.3.000035

[13] Shepp, L.A. and Logan, B.F. (1974) The Fourier Reconstruction of a Head Section. IEEE Transactions on Nuclear Science, 21, 21-43. https://doi.org/10.1109/TNS.1974.6499235

[14] Chesler, D.A. and Riederer, S.J. (1974) Ripple Suppression during Reconstruction in Transverse Tomography. Physics in Medicine and Biology, 20, 632-636. https://doi.org/10.1088/0031-9155/20/4/011

[15] Murase, K., Tanada, S., Inoue, T., Sugawara, Y. and Hamamoto, K. (1993) Improvement of Brain Single Photon Emission Tomography (SPET) Using Transmission Data Acquisition in a Four-Head SPET Scanner. European Journal of Nuclear Medicine, 20, 32-38. https://doi.org/10.1007/BF02261243

Submit or recommend next manuscript to SCIRP and we will provide best service for you:

Accepting pre-submission inquiries through Email, Facebook, LinkedIn, Twitter, etc. A wide selection of journals (inclusive of 9 subjects, more than 200 journals)

Providing 24-hour high-quality service

User-friendly online submission system

Fair and swift peer-review system

Efficient typesetting and proofreading procedure

Display of the result of downloads and visits, as well as the number of cited articles

Maximum dissemination of your research work

Submit your manuscript at: http://papersubmission.scirp.org/

Or contact ojmi@scirp.org 